\title{
Summary of SUSY constraints from ATLAS using the phenomenological MSSM
}

\author{
William James FAWCETT*, on behalf of the ATLAS Collaboration \\ University of Oxford (GB) \\ E-mail: william.fawcett@cern.ch
}

\begin{abstract}
A summary of the ATLAS collaboration's sensitivity to $R$-parity conserving supersymmetry is presented. A total of 22 beyond-the-Standard-Model searches for exotic particles, heavy Higgs bosons and supersymmetric partner particles have been combined to test the validity of a large class of supersymmetric scenarios. In this selection of models, all relevant dark matter, heavy flavour and precision electroweak measurements have been taken into account. The results of these searches are interpreted in the 19-parameter phenomenological-MSSM, and allow one to see where there is room for new physics still to be hiding, in some cases at surprisingly low masses. This is the first time such a large-scale study of this kind has been attempted by an LHC collaboration.
\end{abstract}

XXVII International Symposium on Lepton Photon Interactions at High Energies 17-22 August 2015

Ljubljana, Slovenia

\footnotetext{
* Speaker.
} 


\section{Introduction}

The LHC experiments typically present their results for supersymmetry (SUSY) searches in terms of low-scale simplified models. These models typically contain two or three free parameters and can fail to capture the complexity of more realistic SUSY scenarios, in which many different sparticles have competing production and decay mechanisms. On the other hand, the full minimal supersymmetric extension to the Standard Model (MSSM) has over 100 parameters, making it too complex to explore directly. To study the impact of LHC Run 1 on the supersymmetric parameter space in a more general way, 22 ATLAS searches (listed in Ref. [1]) for beyond-the-StandardModel (BSM) physics have been combined and the results interpreted in the context of the 19 parameter $R$-parity conserving phenomenological-MSSM (pMSSM) [2]. This approach has several advantages over that of the simplified model case in that the full MSSM sparticle spectrum is available, and due to the larger parameter space, correlations between different types of search can be studied. Throughout, the lightest supersymmetric particle (LSP) is the lightest neutralino, $\tilde{\chi}_{1}^{0}$.

\section{Model generation and classification}

The first stage of the analysis was to generate viable models. Results from heavy flavour measurements (such as the branching ratio of $B_{s} \rightarrow \mu^{+} \mu^{-}$), non-collider constraints (such as the dark matter relic density) and precision electroweak measurements (such as $g_{\mu}-2$ ) were incorporated. In order to sample the pMSSM parameter space, approximately $500 \times 10^{6}$ points (sets of parameters) were randomly scanned with a flat prior in each parameter. The range of each parameter was restricted, with the lower limit chosen to avoid experimental constraints, and the upper limit chosen to give a high density of points with masses in reach of LHC experiments (for a list of parameters and scan ranges see Ref. [1]). Importantly, all sparticle masses were limited to be less than $4 \mathrm{TeV}$.

After applying these constraints approximately $310 \times 10^{3}$ models remained. The next step of the analysis was to classify these models as excluded by the ATLAS searches or not. Firstly, around $10 \%$ of these models had a total production cross-section so small that it would have been impossible to exclude them with LHC searches. The remaining models were simulated at particle level, resulting in the generation of about 33 billion events, and yields were calculated with ATLAS analysis software. Models with yields much greater (less) than model independent-limits were classified as (not) excluded. For around 5\% of models, yields were similar to model independent limits and so these models underwent detector simulation and reconstruction to take into account particle interactions with the ATLAS detector, and the detector's response. These models were then treated with the CLs prescription, being classified as excluded if $p<0.05$ (i.e. excluded at $95 \%$ CLs). Full details of this method can be found in Ref. [1].

\section{Results and Discussion}

The impact of the combined ATLAS searches is presented as projections of the 19-parameter pMSSM onto two-dimensional planes; the most relevant parameters typically being sparticle masses. The reach of the combination of searches in the $\tilde{g}_{-} \tilde{\chi}_{1}^{0}$ mass plane is shown in Figure 1(a). Each bin of the plot contains many models, and so the colour code shows the fraction of model points 


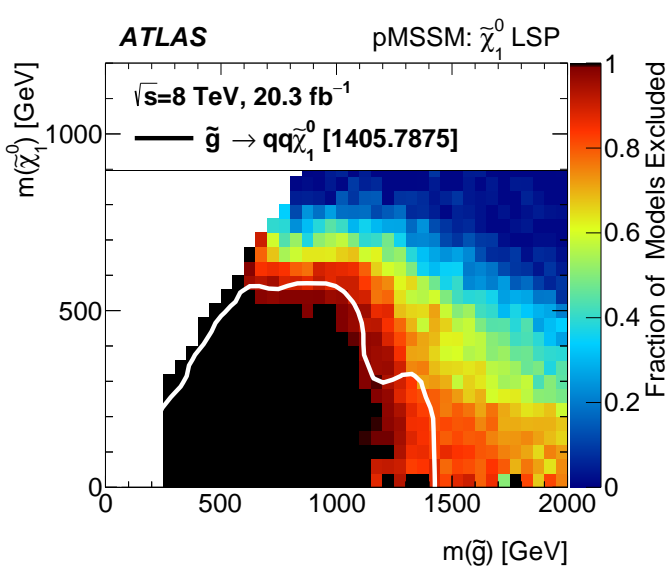

(a)

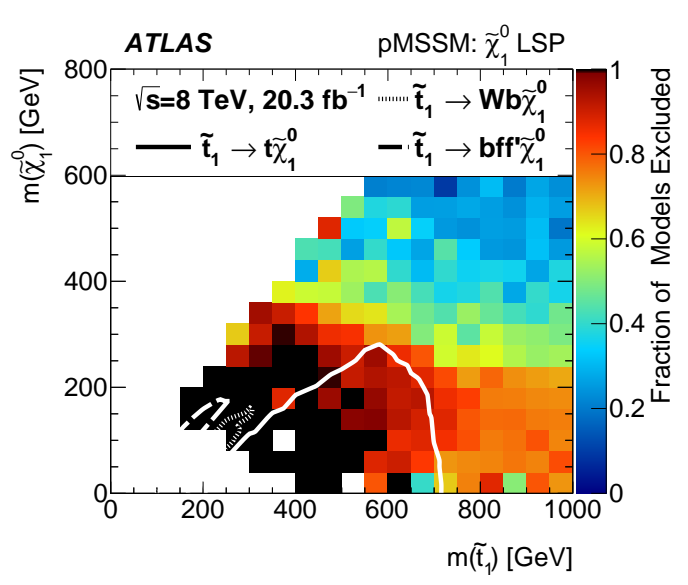

(b)

Figure 1: The impact of the combined ATLAS searches, projected onto the $\tilde{g}-\tilde{\chi}_{1}^{0}$ mass plane (left) and the $\tilde{t}_{1}-\tilde{\chi}_{1}^{0}$ mass plane (right). The colour code shows the fraction of models excluded in each bin by the ATLAS searches, and the white lines are simplified model limit contours as described in the text. Figures taken from Ref. [1].

that have been excluded in each bin (the fraction is relative to the total number of models surviving the non-ATLAS constraints). As one would expect, light gluinos are robustly constrained by the ATLAS searches and no models with $m(\tilde{g})<700 \mathrm{GeV}$ remain, whereas at larger gluino masses the fraction of model points excluded is reduced. Additionally, it is instructive to compare these observed pMSSM exclusions to the observed limits obtained from previous searches. Overlaid on Figure 1(a), the white line is the simplified model limit contour (at 95\% CL) from the 0-lepton 2-6 jets $+E_{\mathrm{T}}^{\mathrm{miss}}$ search [3], which assumes only gluinos are kinematically accessible, and decay directly to the neutralino with a $100 \%$ branching fraction $\left(\tilde{g} \rightarrow q \bar{q}_{1}^{0}\right)$. Given that much more complex decay modes are possible in the pMSSM, it is encouraging that the simplified model limit has a generally good congruence with the combined exclusion in this plane, and it roughly encircles the black region (black represents bins in which $100 \%$ of points have been excluded). In total, around $40 \%$ of the $310 \times 10^{3}$ models were excluded by the ATLAS analyses, although it should be noted this percentage depends strongly on how the parameter space was sampled.

Reference [1] also explores the impact of ATLAS searches on third-generation squarks, which are of particular phenomenological interest due to their relationship with the naturalness problem associated with the mass of the Higgs boson. The fraction of models excluded as projected onto the $\tilde{t}_{1}-\tilde{\chi}_{1}^{0}$ mass plane is shown in Figure 1(b). One observes that most models with $m\left(\tilde{t}_{1}\right)<400 \mathrm{GeV}$ are excluded by ATLAS. Three separate simplified model limit contours have also been superimposed, each assuming direct production of the top squark with a different decay mode for each case. The solid line is the limit for the case $\tilde{t}_{1} \rightarrow t \tilde{\chi}_{1}^{0}$ from Ref. [4] whereas the dashed lines assume $\tilde{t}_{1} \rightarrow W b \tilde{\chi}_{1}^{0}$ and $\tilde{t}_{1} \rightarrow b f f^{\prime} \tilde{\chi}_{1}^{0}$ separately, from Ref. [5]. Overall Figure 1(b) shows that top squark masses are not as tightly constrained as in the simplified model case, however in the compressed region (where the mass splitting between the top squark and neutralino is small) the simplified model limits are an underestimate of the reach of ATLAS searches. 


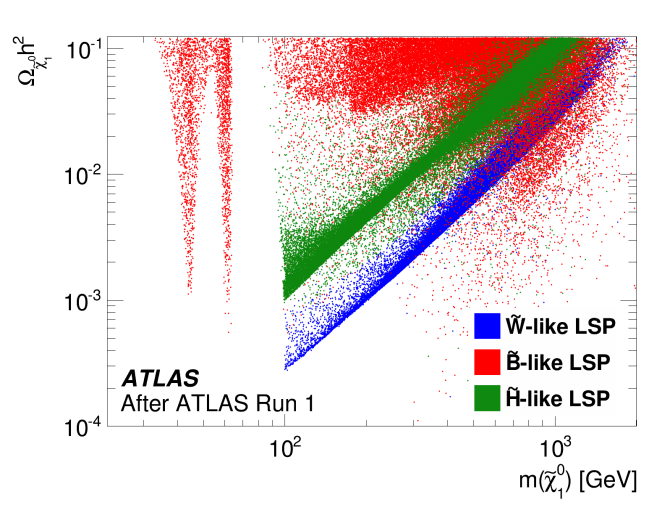

(a)

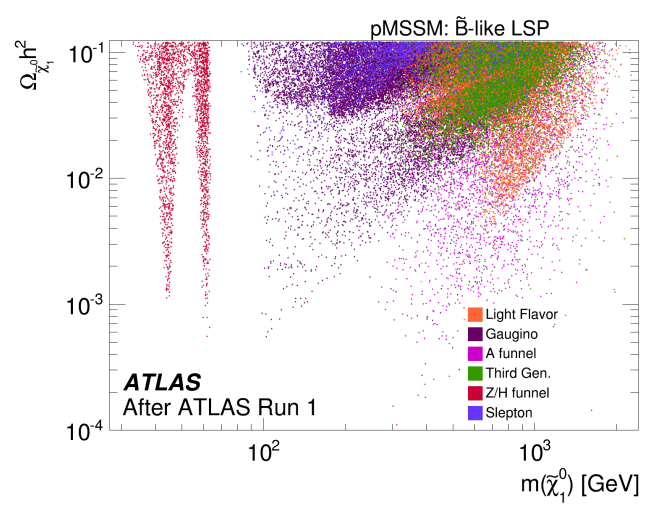

(b)

Figure 2: Density of pMSSM points that were not excluded by ATLAS Run 1 in the plane showing neutralino mass versus dark matter relic density. The left plot colours models according to the dominant component of the LSP. The right plot colours models according to the annihilation mechanisms for models with a dominantly bino LSP. Figures taken from Ref. [1].

As the neutralino is assumed to be a stable, neutral and weakly interacting particle it provides an ideal dark-matter candidate. In principle, the neutralino can be a mixture of bino, wino and Higgsino components. However, for the models considered here the LSP is typically dominated by a particular type, with over $87 \%$ of models having a LSP which is at least $90 \%$ pure. The nature of the neutralino has a strong influence on its contribution to the expected dark matter relic density, $\Omega_{\tilde{\chi}_{1}^{0}} h^{2}$, and this is shown for models eluding ATLAS searches in Figure 2(a). For models with a wino- or higgsino-dominated LSP there is an approximate proportionality that holds for most models, being $\Omega_{\tilde{\chi}_{1}^{0}} h^{2} \propto m\left(\tilde{\chi}_{1}^{0}\right)^{2}$, however the picture is very different for models with a bino-dominated LSP. The structures seen in Figure 2(a) are related to the different annihilation mechanisms of neutralinos, as explained by Figure 2(b). Efficient annihilation, and the subsequent reduction in neutralino relic density, is important as models with a bino-dominated LSP typically produce more dark matter than has been observed by astrophysical experiments. Two examples of mechanisms to reduce the relic density include resonant annihilation of bino-models with mass approximately half that of either the Higgs or $Z$ boson $\left(\tilde{\chi}_{1}^{0} \tilde{\chi}_{1}^{0} \rightarrow Z / h\right)$, the so-called "Funnel" regions, and also co-annihilation of a neutralino and another sparticle (with a sufficiently small mass difference), for example $\tilde{\chi}_{1}^{0} \tilde{\tau} \rightarrow \tau \gamma$.

Indirect searches for new physics can also constrain the SUSY parameter space. If the neutralino is sufficiently light then the decay $h \rightarrow \tilde{\chi}_{1}^{0} \tilde{\chi}_{1}^{0}$ can occur, which has the signature of a Higgs boson decaying invisibly in the ATLAS detector. The distribution of $\operatorname{BR}\left(h \rightarrow \tilde{\chi}_{1}^{0} \tilde{\chi}_{1}^{0}\right)$ before and after the ATLAS searches is shown in Figure 3 for models with $m\left(\tilde{\chi}_{1}^{0}\right)<65 \mathrm{GeV}$. ATLAS has recently set a limit on this branching ratio [6], and this has been overlaid on the figure. The comparison shows how this type of measurement can be complementary to direct searches for BSM physics. 


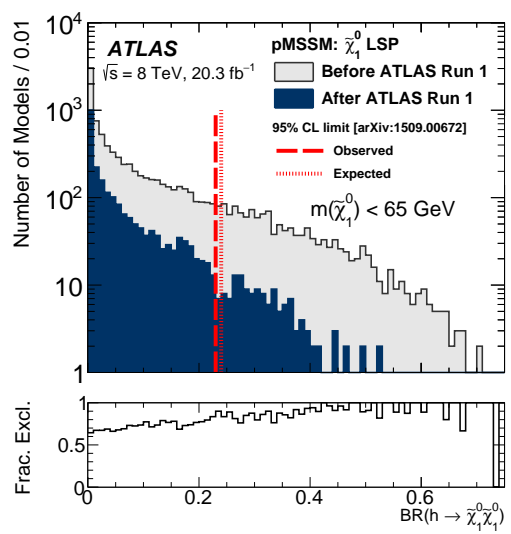

Figure 3: Distribution of $\operatorname{BR}\left(h \rightarrow \tilde{\chi}_{1}^{0} \tilde{\chi}_{1}^{0}\right)$ for model points, before and after the combined ATLAS searches. Only models with $m\left(\tilde{\chi}_{1}^{0}\right)<65 \mathrm{GeV}$ have been plotted. Figure taken from Ref. [1].

\section{Summary}

The ATLAS Collaboration has performed many searches for SUSY and other BSM scenarios with data from Run 1 of the LHC. The results of these searches are usually interpreted by setting limits on sparticle masses in the context of simplified models, however in the broader MSSM, it is less clear as to what sparticle masses have been excluded. A combination of 22 ATLAS searches has been performed to provide a more general statement as to what sparticle masses have been excluded. From an initial random sampling of $500 \times 10^{6}$ pMSSM points generated from the 19parameter pMSSM, about $310 \times 10^{3}$ models are selected, each of which satisfies constraints from previous collider searches, electroweak measurements and dark matter constraints. These models are classified as excluded, or not, by the combined ATLAS searches. The results are interpreted in sparticle mass planes, dark matter observables and other observables such as $\operatorname{BR}\left(h \rightarrow \tilde{\chi}_{1}^{0} \tilde{\chi}_{1}^{0}\right)$.

\section{References}

[1] ATLAS Collaboration, Summary of the ATLAS experiment's sensitivity to supersymmetry after LHC Run 1 -interpreted in the phenomenological MSSM. Submitted to JHEP [arXiv:1508.06608]

[2] M. Cahill-Rowley, J. Hewett, A. Ismail and T. Rizzo, More energy, more searches, but the phenomenological mssm lives on. Phys. Rev. D 88 (2013) 035002. [arXiv:1211.1981]

[3] ATLAS Collaboration, Search for squarks and gluinos with the ATLAS detector in final states with jets and missing transverse momentum using $\sqrt{s}=8 \mathrm{TeV}$ proton-proton collision data. JHEP 09 (2014) 176. [arXiv:1405.7875]

[4] ATLAS Collaboration, ATLAS Run 1 searches for direct pair production of third-generation squarks at the Large Hadron Collider. Submitted to Eur. Phys. J. [arXiv:1506.08616]

[5] ATLAS Collaboration, Search for top squark pair production in final states with one isolated lepton, jets, and missing transverse momentum in $\sqrt{s}=8 \mathrm{TeV}$ pp collisions with the ATLAS detector. JHEP 11 (2014) 118. [arXiv:1407.0583]

[6] ATLAS Collaboration, Constraints on new phenomena via Higgs boson couplings and invisible decays with the ATLAS detector. Submitted to JHEP. [arXiv:1509.00672] 